\title{
Models of true arithmetic are integer parts of models of real exponentiation
}

\author{
MERLIN CARL \\ LOTHAR SEBASTIAN KRAPP
}

\begin{abstract}
Exploring further the connection between exponentiation on real closed fields and the existence of an integer part modelling strong fragments of arithmetic, we demonstrate that each model of true arithmetic is an integer part of a real closed exponential field that is elementarily equivalent to the real numbers with exponentiation and that each model of Peano arithmetic is an integer part of a real closed field that admits an isomorphism between its ordered additive and its ordered multiplicative group of positive elements. Under the assumption of Schanuel's Conjecture, we obtain further strengthenings for the last statement.
\end{abstract}

2020 Mathematics Subject Classification $03 \mathrm{C} 64$ (primary); 06A05, 03C60, 03H15, 12J15, 12L12 (secondary)

Keywords: true arithmetic, Peano arithmetic, integer parts, real exponentiation, exponential fields

\section{Introduction}

An integer part (IP) of a real closed field $K$ is a discretely ordered subring $R \subseteq K$ with minimal positive element 1 such that, for any $x \in K$, there is a unique element $\lfloor x\rfloor \in R$ with $\lfloor x\rfloor \leq x<\lfloor x\rfloor+1$ (see eg Shepherdson [21]). In [4, §5], it was shown by D'Aquino, Knight and Starchenko that a countable real closed field $K$ has an IP modelling PA if and only if $K$ is recursively saturated. Marker, Schmerl and Steinhorn [18, § 1] gave a counterexample in the uncountable case: they constructed $\aleph_{1}$-saturated real closed Hahn series fields $K$ and showed that an exponential function on an IP of $K$ modelling PA can be lifted to an exponential on $K$. Since by F.-V. Kuhlmann, S. Kuhlmann and Shelah [14] such an exponential on $K$ cannot exist, $K$ does not admit an IP modelling PA. The methods of [18] were refined in Carl, D' Aquino and S. Kuhlmann [3] to the theorem that real closed fields with IPs modelling $I \Delta_{0}+$ EXP always allow a weak form of exponentiation known as 'left-exponentiation', that is, an isomorphism from an additive group complement of the valuation ring for the 
natural valuation to a multiplicative group complement of the positive group of units (see S. Kuhlmann [15, page 24]). It is then natural to ask what influence a model of arithmetic has on the spectrum of real closed fields of which it is an IP.

We show that models of true arithmetic are always IPs of real closed fields that are very similar to the real numbers with exponentiation in a model theoretic sense: Namely, let us say-following the usual terminology-that a function $E: K \rightarrow K$ on a real closed field $K$ is an exponential if it defines an isomorphism between $(K,+, 0,<)$ and $\left(K^{>0}, \cdot, 1,<\right)$. The structure $(K,+, \cdot, 0,1,<, E)$ is then called a real closed exponential field. Furthermore, let us say that a real closed exponential field $(K,+, \cdot, 0,1,<, E)$ is a model of real exponentiation if it is elementarily equivalent to $\mathbb{R}_{\exp }=\left(\mathbb{R},+, \cdot, 0,1,<, 2^{x}\right)$, where $2^{x}$ denotes the usual exponentiation with base 2 on the real numbers. Then each model of true arithmetic, ie of $\operatorname{Th}(\mathbb{N},+, \cdot, 0,1,<)$, is an IP of a model of real exponentiation. ${ }^{1}$ We also show that this fails if one replaces true arithmetic with bounded arithmetic $\left(I \Delta_{0}\right)$. We conjecture that Peano arithmetic is actually enough to achieve our results and obtain the following partial result: if $(M,+, \cdot, 0,1,<) \models \mathrm{PA}$, then it is an IP of a real closed exponential field $\left(K_{M},+, \cdot, 0,1,<, \exp _{M}\right)$. Moreover, under the assumption of Schanuel's Conjecture, if this real closed exponential field is model complete, then it satisfies the existential theory of $\mathbb{R}_{\text {exp }} \cdot{ }^{2}$ We do not know where the exact benchmark is.

Our result can be seen as a further variation of one direction of a well-known theorem of Shepherdson [21], according to which each model of open induction-Peano arithmetic with induction restricted to open (ie quantifier-free) formulas - is an IP of a real closed field. The result of Carl, D'Aquino and S. Kuhlmann [3] mentioned above implies that each model of $I \Delta_{0}+$ EXP is an exponential IP of a real closed left-exponential field. Finally, by Krapp [13, Theorem 7.26] any model of PA is an IP of a real closed exponential field. Together with the theorems proved in this paper, we hence get the following picture:

- $M \models I$ Open $\rightarrow M$ is an IP of a real closed field.

- $M \models I \Delta_{0}+\mathrm{EXP} \rightarrow M$ is an (exponential) IP of a real closed field admitting left-exponentiation.

\footnotetext{
${ }^{1}$ By saying that a discretely ordered semiring $M$ is an IP of some real closed field $K$, we mean—by abuse of terminology—that the corresponding discretely ordered ring $M \cup(-M)$ is an IP of $K$.

${ }^{2}$ Schanuel's Conjecture is intimately linked to the decidability of the theory of real exponentiation and of its existential subtheory. Macintyre [17] deals with these links and draws further connections to computable models of real exponentiation (see [17, Section 3]). We point out that in contrast to Macintyre's deliberately not specified notion of computability, we deal with reals which are arithmetically definable.
} 
- $M \models \mathrm{PA} \rightarrow M$ is an IP of a real closed exponential field.

- $M \models \mathrm{Th}(\mathbb{N}) \rightarrow M$ is an IP of a model of real exponentiation.

Notation 1.1 We denote by $\mathbb{N}$ the set of natural numbers with 0 . If $\vec{v}=\left(v_{1}, \ldots, v_{n}\right)$ and $M$ is a set, then $\vec{v} \in M$ means that $\vec{v}$ is a tuple of elements of $M$. The language of Peano arithmetic is denoted by $\mathcal{L}_{\mathrm{PA}}$ and consists of the binary function symbols + and $\cdot$, the constant symbols 0 and 1 and the binary relation symbol $<$. The language $\mathcal{L}_{\text {exp }}$ of exponential rings is obtained by adding a unary function symbol $E$ to $\mathcal{L}_{\mathrm{PA}}$. If no confusion is likely to arise, we denote an $\mathcal{L}_{\mathrm{PA}}-$ structure $(M,+, \cdot, 0,1,<)$ simply by $M$ and an $\mathcal{L}_{\exp }$-structure $(K,+, \cdot, 0,1,<, E)$ simply by $(K, E)$. Base $b$ exponentiation on $\mathbb{R}$ is denoted by $b^{x}$ and the standard exponential function $\mathrm{e}^{x}$ on $\mathbb{R}$ by $\exp$. If $M$ is a commutative semiring without zero divisors, then $\mathbb{Z}_{M}$ denotes $-M \cup M$ and $\mathbb{Q}_{M}$ denotes the fraction field ff( $\left.\mathbb{Z}_{M}\right)$. We call $\mathbb{Z}_{M}$ the set of $M$-integers and $\mathbb{Q}_{M}$ the set of $M$-rationals.

\section{The real closed field of $M$-reals}

We now define an analogue of the real numbers for an arbitrary model of Peano arithmetic. In the context of second-order arithmetic and reverse mathematics, real numbers are usually modelled as Cauchy sequences of rational numbers, see eg Simpson [22, Chapter 1], and we follow this approach here.

Throughout Section 2 and Section 3, we fix a model $M \models \mathrm{PA}$. We will denote the real closed field of $M$-reals of which $M$ will be an IP by $K_{M}$. It is crucial to point out that the construction of a real closed field in [22] takes place in models of second-order arithmetic-more specifically, in models of the system $\mathrm{ACA}_{0}$ (arithmetical comprehension axiom) - rather than in first-order models of PA. However, as emphasised in [22, Remark I.3.3], the first-order part of $\mathrm{ACA}_{0}$ is just PA. Since in our model theoretical context it is more suitable to stay in the first-order language $\mathcal{L}_{\mathrm{PA}}$, we apply the results of [22] directly to $M$ and point out where special care is needed.

Our first aim is to establish a way to quantify in $M$ over the set of $M$-integers $\mathbb{Z}_{M}$ and the set of $M$-rationals $\mathbb{Q}_{M}$, ie we want to confer meaning to expressions like $M=\left(\exists x \in \mathbb{Q}_{M}\right) \varphi(x)$ or $M=\left(\forall x \in \mathbb{Q}_{M}\right)\left(\exists y \in \mathbb{Z}_{M}\right) \psi(x, y)$, where $\varphi$ and $\psi$ are $\mathcal{L}_{\mathrm{PA}}-$ formulas. This can easily be done by representing the set of $M$-integers and the 
set of $M$-rationals as follows:

$$
\begin{aligned}
& \mathbb{Z}_{M}=\left\{p_{1}-p_{2}: p_{1}, p_{2} \in M\right\} \\
& \mathbb{Q}_{M}=\left\{\frac{p_{1}-p_{2}}{q_{1}-q_{2}}: p_{1}, p_{2}, q_{1}, q_{2} \in M, q_{1}-q_{2} \neq 0\right\}
\end{aligned}
$$

Hence, any $M$-integer associates to an equivalence class of pairs $\left(p_{1}, p_{2}\right) \in M^{2}$ and any $M$-rational to an equivalence class of quadruples $\left(p_{1}, p_{2}, q_{1}, q_{2}\right) \in M^{4}$.

Atomic formulas in $\mathcal{L}_{\mathrm{PA}}$ are equivalent over PA to formulas of the form $p(\vec{x})=q(\vec{x})$ or $p(\vec{x})<q(\vec{x})$, where $p$ and $q$ are polynomials with coefficients in $\mathbb{N}$. Moreover, equations and inequations in $\mathbb{Z}_{M}$ involving the arithmetic operation of subtraction are always equivalent to an expression without subtraction, eg $p_{1}-p_{2}<0$ can be expressed equivalently by $p_{1}<p_{2}$. This way, we can confer meaning to quantification over $\mathbb{Z}_{M}$ within $M$ (also using parameters from $\mathbb{Z}_{M}$ and the arithmetic operation of subtraction). For instance, we interpret the expression $\exists\left(y \in \mathbb{Z}_{M}\right) x-y>-2 x^{2}+x y$ as the $\mathcal{L}_{\mathrm{PA}}-$ formula $\exists y\left(2 x^{2}+x>x y+y \vee 2 x^{2}+x y+x+y>0\right)$. In a similar way, we can quantify in $M$ over the set of $M$-rationals $\mathbb{Q}_{M}$ and use parameters from $\mathbb{Q}_{M}$ by clearing fractions in the polynomials which appear in the atomic formulas. For instance, $\exists\left(x \in \mathbb{Q}_{M}\right) x^{2}-\frac{1}{2}>x$ stands for $\exists\left(a \in \mathbb{Z}_{M}\right) \exists(b>0) 2 a^{2}-b^{2}>2 a b$.

Quantification over the set of $M$-rationals within $M$ lays the foundation for the notion of rational $M$-Cauchy sequences we will introduce in the following. To ease the notation, from now on we consider $\mathbb{Q}_{M}$ rather than $M$ as the domain over which we work. Due to the explanations above, for each $\mathcal{L}_{\mathrm{PA}}-$ formula $\varphi(\vec{t})$, there is an $\mathcal{L}_{\mathrm{PA}}$-formula $\psi(\vec{x}, \vec{y}, \vec{u}, \vec{v})$ (where all tuples $\vec{t}, \vec{x}, \vec{y}, \vec{u}$ and $\vec{v}$ have the same lengths) such that for any $\vec{q} \in \mathbb{Q}_{M}$, there exist $\vec{a}, \vec{b}, \vec{c}, \vec{d} \in M$ such that:

$$
\mathbb{Q}_{M}=\varphi(\vec{q}) \text { if and only if } M=\psi(\vec{a}, \vec{b}, \vec{c}, \vec{d})
$$

Hence, by abuse of notation, from now on also all quantifiers are assumed to be over $\mathbb{Q}_{M}$ unless they are explicitly pointed out to be over $M$.

Rational $M$-Cauchy sequences are Cauchy sequences in $\mathbb{Q}_{M}$ definable ${ }^{3}$ over $\mathbb{Q}_{M}$. As we pointed out at the beginning of this section, the results we apply regard second-order models of $\mathrm{ACA}_{0}$. These can be obtained from models of PA by restricting second-order quantification to arithmetically definable sets (see [22, page 8]). In other words, the results regarding $\mathrm{ACA}_{0}$ we wish to use still apply to any model of PA as long as the sets involved are definable. Hence, we regard the set of $M$-reals $K_{M}$ as the set of equivalence classes of Cauchy sequences in $\mathbb{Q}_{M}$ which are definable over $\mathbb{Q}_{M}$. We make this precise in the following.

\footnotetext{
${ }^{3}$ Throughout this paper, "definable" always means "definable with parameters".
} 
A rational $M$-sequence is a function $a: M \rightarrow \mathbb{Q}_{M}$ which is definable over $\mathbb{Q}_{M}$. As usual, we denote $a(n)$ by $a_{n}$ and $a$ also by $\left(a_{n}\right)_{n \in M}$.

Let $a$ be a rational $M$-sequence. We say that $a$ is a rational $M$-Cauchy sequence if the following holds:

$$
\mathbb{Q}_{M}=\forall(\varepsilon>0) \exists(N \in M) \forall\left(m, n \in M^{>N}\right)\left|a_{n}-a_{m}\right|<\varepsilon
$$

We define an equivalence relation $\sim$ on the set of rational $M$-Cauchy sequences by $a \sim b$ if the following holds:

$$
\mathbb{Q}_{M}=\forall(\varepsilon>0) \exists(N \in M) \forall\left(n \in M^{>N}\right)\left|a_{n}-b_{n}\right|<\varepsilon
$$

The set of all equivalence classes of rational $M$-Cauchy sequences is denoted by $K_{M}$. We call $K_{M}$ the set of $M$-reals. We interpret the symbols of $\mathcal{L}_{\mathrm{PA}}$ in $K_{M}$ as follows (where $[a]$ stands for the $\sim$-equivalence class of a rational $M$-Cauchy sequence):

- $[a]+[b]=[a+b]$

- $[a] \cdot[b]=[a \cdot b]$

- $[a]<[b]$ if $\mathbb{Q}_{M} \models \exists(\delta>0) \exists(N \in M) \forall\left(n \in M^{>N}\right) a_{n}+\delta<b_{n}$

If it is clear from the context, then we also denote an $M$-real $[a]$ simply by $a$ and a rational $M$-sequence $\left(a_{n}\right)_{n \in M}$ by $\left(a_{n}\right)$ or $a_{n}$. We also say that a rational $M$-Cauchy sequence $\left(a_{n}\right)_{n \in M}$ converges to $a$ if $a=\left[\left(a_{n}\right)_{n \in M}\right]$, and we write $\lim _{n \rightarrow \infty} a_{n}=a$. For any $q \in \mathbb{Q}_{M}$, we also denote the $M$-real given by the equivalence class of the $M$-sequence which is constantly $q$ by $q$. In particular, this gives suitable interpretations of the symbols 0 and 1 in $K_{M}$. By [22, Theorem II.4.5], $K_{M}$ is an ordered field. Moreover, $\mathbb{Q}_{M}$ is an ordered subfield of $K_{M}$ (via the identification described above), and thus we can also consider $M$ and $\mathbb{Z}_{M}$ as subsets of $K_{M}$ such that all arithmetic operations as well as the order relation are preserved.

Since $K_{M}$ ranges over equivalence classes of definable functions in $\mathbb{Q}_{M}$, we cannot quantify in $\mathbb{Q}_{M}$ over elements of $K_{M}$. However, we can confer meaning to formulas using finitely many parameters from $K_{M}$. For instance, if $a, b \in K_{M}$, then an expression of the form $\mathbb{Q}_{M} \models \exists x a<x$ stands for $\mathbb{Q}_{M}=\exists x \exists(\varepsilon>0) \exists(N \in M) \forall\left(n \in M^{>N}\right) a_{n}+\varepsilon<x$, where $\left(a_{n}\right)$ is a rational $M$-sequence converging to $a$; and $\mathbb{Q}_{M} \models \exists x a+x=b$ is short for $\mathbb{Q}_{M}=\exists x \neg(a+x-b)^{2}>0$.

Theorem 2.1 The ordered field of $M$-reals $K_{M}$ is real closed. Moreover, $M$ is cofinal in $K_{M}$, ie for any $a \in K_{M}$, there exists $n \in M$ such that $a<n$.

Proof That $K_{M}$ is real closed is proved in [22, Corollary II.6.7]. The property of $M$ to be cofinal in $K_{M}$ is proved in [22, Theorem II.4.5]. 
Corollary 2.2 The ring of $M$-integers $\mathbb{Z}_{M}$ is an IP of $K_{M}$. Hence, in particular, $\mathbb{Q}_{M}$ is dense in $K_{M}$.

Proof Let $a \in K_{M}$. Since $\mathbb{Z}_{M}$ is a discretely ordered ring with 1 as least positive element, we only need to show that $b \leq a<b+1$ for some $b \in \mathbb{Z}_{M}$. By Theorem 2.1, the definable set $\left\{n \in M: \mathbb{Q}_{M}\right\} \models|a|<n$ is non-empty. Thus, it must have a least element $m$. We obtain $m-1 \leq|a|<m$, giving the required integer part of $a$.

Notation 2.3 Let $\varphi(n, y, \vec{v})$ be an $\mathcal{L}_{\mathrm{PA}}-$ formula and let $\vec{p} \in \mathbb{Q}_{M}$ such that $\varphi(n, y, \vec{p})$ defines an $M$-real ${ }^{4}$. Then this $M$-real is denoted by $x_{\varphi}^{\vec{p}}$.

We are almost exclusively interested in arithmetical formulas $\varphi(n, y, \vec{v})$ that define an $M$-real for every model $M$ of PA and any tuple $\vec{p}$ of elements of $\mathbb{Q}_{M}$, ie such that:

$$
\mathbb{Q}_{M} \models \forall \vec{v} ‘ \varphi(n, y, \vec{v}) \text { defines a rational } M \text {-Cauchy sequence.' }
$$

Let us call such a formula $\varphi$ a safe formula.

In later sections, we will assume that all occurring $\mathcal{L}_{\mathrm{PA}}-$ formulas (with at least two free variables) are of this kind, unless stated otherwise. However, we have to ensure that by this restriction we do not lose any $M$-reals.

Lemma 2.4 For any $\mathcal{L}_{\mathrm{PA}}-$ formula $\varphi(n, y, \vec{v})$ there exists an $\mathcal{L}_{\mathrm{PA}}-$ formula $\varphi^{\prime}(n, y, \vec{v})$ such that the following is a theorem of PA: for every parameter $\vec{p} \in \mathbb{Q}_{M}$, if $\varphi(n, y, \vec{p})$ defines a rational $M$-Cauchy sequence, then $\varphi(n, y, \vec{p})$ and $\varphi^{\prime}(n, y, \vec{p})$ define the same sequence, otherwise $\varphi^{\prime}(n, y, \vec{p})$ defines the constant 0 function. Thus, if $x$ is an $M$-real, then there exist a safe formula $\varphi$ and a tuple $\vec{p} \in \mathbb{Q}_{M}$ such that $x=x_{\varphi}^{\vec{p}}$.

Proof We abbreviate by $\operatorname{conv}(\varphi, \vec{v})$ the $\mathcal{L}_{\mathrm{PA}}-$ formula expressing that $\varphi(n, y, \vec{v})$ defines a rational Cauchy sequence $\left(a_{n}\right)$. Now let $\varphi^{\prime}(n, y, \vec{v})$ be the $\mathcal{L}_{\mathrm{PA}}-$ formula:

$$
[\operatorname{conv}(\varphi, \vec{v}) \rightarrow \varphi(n, y, \vec{v})] \wedge[\neg \operatorname{conv}(\varphi, \vec{v}) \rightarrow y=0]
$$

Then for any parameter $\vec{p}$, if $\varphi(n, y, \vec{p})$ defines a rational Cauchy sequence, then $\varphi^{\prime}(n, y, \vec{p})$ defines the same sequence, and otherwise $\varphi^{\prime}(n, y, \vec{p})$ defines the function mapping each $n$ to 0 .

\footnotetext{
${ }^{4}$ To be precise, an $\mathcal{L}_{\mathrm{PA}}$-formula does not define an $M$-real but only a rational $M$-Cauchy sequence representing this $M$-real. However, we use this abuse of terminology, as it should be clear from the context whether an $M$-real or a corresponding rational $M$-Cauchy sequence is meant.
} 


\section{Analysis on $K_{M}$}

\subsection{Definable functions}

Before we introduce an exponential function, we consider general analysis on $K_{M}$. To this purpose, we need to introduce the notion of definable functions on $K_{M}$. If properties of these functions are to be preserved between fields of $M$-reals arising from different models of PA, then these will have to be sufficiently explicitly definable in PA. This is made precise in the following.

Definition 3.1 Let $m \in \mathbb{N}^{>0}$ and let $f: K_{M}^{m} \rightarrow K_{M}$. Then $f$ is $M$-definable if there exist an $\mathcal{L}_{\mathrm{PA}^{-}}$formula $\psi\left(n, y, \vec{v}, X_{1}, \ldots, X_{m}\right)$ and a tuple $\vec{p} \in \mathbb{Q}_{M}$ such that for any rational $M$-Cauchy sequences $a_{n}^{(1)}, \ldots, a_{n}^{(m)}$ we have that

$$
\psi\left(n, y, \vec{p}, a_{n}^{(1)}, \ldots, a_{n}^{(m)}\right)
$$

defines the $M$-real $f\left(a^{(1)}, \ldots, a^{(m)}\right)$, where $a^{(i)}=\lim _{n \rightarrow \infty} a_{n}^{(i)}$. We then say that $\psi\left(n, y, \vec{p}, X_{1}, \ldots, X_{m}\right)$ defines $f$. Denote by $\operatorname{Def}^{n}(M)$ the set of $n$-ary $M$-definable functions and let $\operatorname{Def}(M)=\bigcup_{i \in \mathbb{N}>0} \operatorname{Def}^{i}(M)$.

Proposition 3.2 The set $\operatorname{Def}(M)$ contains all constant functions and is closed under composition.

Proof We prove both statements for functions $f: K_{M} \rightarrow K_{M}$, as the multivariable case works similarly. Let $\varphi(n, y, \vec{v})$ be a safe $\mathcal{L}_{\mathrm{PA}}-$ formula, let $\vec{p} \in \mathbb{Q}_{M}$ and set $c=x_{\varphi}^{\vec{p}}$. Then the formula $\psi(n, y, \vec{p}, X)$ given by $n \in M \wedge \varphi(n, y, \vec{p})$ defines $f: K_{M} \rightarrow K_{M}, u \mapsto c$.

Now let $f, g \in \operatorname{Def}^{1}(M)$ and let $\psi_{f}(n, y, \vec{p}, X)$ and $\psi_{g}(n, y, \vec{p}, X)$ define $f$ and $g$, respectively. Define the $\mathcal{L}_{\mathrm{PA}^{-}}$formula $\psi_{\text {gof }}(n, y, \vec{v}, X)$ as follows:

$$
\forall q\left(\psi_{f}(n, q, \vec{v}, X) \rightarrow \psi_{g}(n, y, \vec{v}, q)\right)
$$

We show that $\psi_{g \circ f}(n, y, \vec{p}, X)$ defines $g \circ f$. Let $a \in K_{M}$ and let $a_{n}$ be a rational $M$-Cauchy sequence with $\lim _{n \rightarrow \infty} a_{n}=a$. Then $\psi_{f}\left(n, y, \vec{p}, a_{n}\right)$ defines $f(a)$. Hence, for any $n \in M$ there is a unique $q_{n} \in \mathbb{Q}_{M}$ such that $\psi_{f}\left(n, q_{n}, \vec{p}, a_{n}\right)$ holds and, moreover, $\lim _{n \rightarrow \infty} q_{n}=f(a)$. Since $\psi_{g}(n, y, \vec{p}, X)$ defines $g$, we obtain that $\psi_{g}\left(n, y, \vec{p}, q_{n}\right)$ defines $g(f(a))$, as required.

Since all ring operations, ie addition, subtraction and multiplication, are clearly $M-$ definable, we obtain the following corollary. 
Corollary 3.3 Let $f_{1}, \ldots, f_{n}$ be $M$-definable continuous functions and let $g \in K\left[x_{1}, \ldots\right.$, $\left.x_{n}\right]$. Then $g\left(f_{1}, \ldots, f_{n}\right)$ is also $M$-definable and continuous.

Proof By Proposition 3.2, $g\left(f_{1}, \ldots, f_{n}\right)$ is $M$-definable. Since in any ordered field the composition of continuous functions is continuous, also $g\left(f_{1}, \ldots, f_{n}\right)$ is continuous.

If a continuous function $f$ is $M$-definable, then we can establish a way to interpret over $\mathbb{Q}_{M}$ formulas in the language $\mathcal{L}_{\mathrm{PA}}$ expanded by $f$ and with finitely many parameters from $K_{M}$. As the multivariable case works similarly, we illustrate this in the single variable case.

Let $f: K_{M} \rightarrow K_{M}$ be continuous and defined by $\psi(n, y, \vec{p}, X)$ for some $\vec{p} \in \mathbb{Q}_{M}$. Let $c \in \mathbb{Q}_{M}$. Then $\psi(n, y, \vec{p}, c)$ defines $f(c)$. Hence, we have that $f(c)>0$ if and only if the following holds in $\mathbb{Q}_{M}$ :

$$
\exists(\varepsilon>0) \exists(N \in M) \forall\left(n \in M^{>N}\right) \forall q(\psi(n, q, \vec{p}, c) \rightarrow q>\varepsilon)
$$

Now let $c \in K_{M}$ be arbitrary and let $c_{n}$ be a rational $M$-Cauchy sequence with $\lim _{n \rightarrow \infty} c_{n}=c$. Then $f(c)>0$ if and only if

$$
\mathbb{Q}_{M} \models \exists(\varepsilon>0) \exists(N \in M) \forall\left(n \in M^{>N}\right) f\left(c_{n}\right)>\varepsilon .
$$

This equivalence follows directly from the continuity of $f$. Finally, $f(c)=0$ holds if and only if $\mathbb{Q}_{M}=\neg(f(c))^{2}>0$.

We have thus overall established a meaningful interpretation of expressions of the form $\mathbb{Q}_{M}=\varphi(\vec{a})$, where $\varphi(\vec{x})$ is a formula in $\mathcal{L}_{\mathrm{PA}}$ expanded by continuous $M$-definable functions, with quantification over $M, \mathbb{Z}_{M}$ as well as $\mathbb{Q}_{M}$, and $\vec{a}$ is a tuple in $K_{M}$.

\subsection{Exponentiation on $K_{M}$}

The main purpose of this subsection is to introduce an exponential $\exp _{M}$ on $K_{M}$, ie an isomorphism from $\left(K_{M},+, 0,<\right)$ to $\left(K_{M}^{>0}, \cdot, 1,<\right)$. Moreover, we establish some analytic results that enable us to quantify in $M$ over $\mathcal{L}_{\exp }$-formulas.

Recall that over PA, the binary exponentiation function $(m, n) \mapsto m^{n}$ is definable. We are interested in base 2 exponentiation, ie the function $2^{x}$ on $M$. In the following, we extend $2^{x}$ to an exponential $\exp _{M}$ with domain $K_{M}$.

For any $n, b, c \in M^{>0}$, consider the set $B=\left\{m \in M: m^{c} \leq 2^{n c+b}\right\}$. Since $1 \in B$, this set is non-empty. Moreover, since $B$ is definable in $M$ and has an upper bound $2^{n+b}$, by overspill we obtain that $B$ has a maximum in $M$. We define:

$$
\operatorname{appr}(n, b, c)=\max B
$$


Let $a \in K_{M}^{>0}$ and let $b_{n}$ and $c_{n}$ be $M$-sequences in $M^{>0}$ such that $a_{n}=b_{n} / c_{n}$ and $a=\lim _{n \rightarrow \infty} a_{n}$. For any $n \in M^{>0}$, let $d_{n}=\operatorname{appr}\left(n, b_{n}, c_{n}\right)$. We define $\exp _{M}(a)$ as:

$$
\exp _{M}(a)=\lim _{n \rightarrow \infty} \frac{d_{n}}{2^{n}}
$$

Moreover, we set $\exp _{M}(0)=1$, and for $a \in K_{M}^{<0}$, we set $\exp _{M}(a)=\exp _{M}(-a)^{-1}$.

Note that $d_{n}$ is the unique element of $M$ satisfying:

$$
\frac{d_{n}^{c_{n}}}{2^{n c_{n}}} \leq 2^{b_{n}}<\frac{\left(d_{n}+1\right)^{c_{n}}}{2^{n c_{n}}}
$$

Hence, for each $n \in M^{>0}$, the $M$-rational $d_{n} / 2^{n}$ is supposed to approximate the value of $2^{b_{n} / c_{n}}$ with an error of at most $1 / 2^{n}$.

In order to show that $\exp _{M}$ is well-defined, one has to verify that for each positive $M$-real $a$ as above, the sequence $d_{n} / 2^{n}$ is a rational $M$-Cauchy sequence, and that two equivalent rational $M$-Cauchy sequences $\left(a_{n}\right)$ and $\left(a_{n}^{\prime}\right)$ produce equivalent rational $M$-Cauchy sequences $d_{n} / 2^{n}$ and $d_{n}^{\prime} / 2^{n}$. To establish that $\exp _{M}$ is indeed an exponential, one has to check the properties of isomorphy from $\left(K_{M},+, 0,<\right)$ to $\left(K_{M}^{>0}, \cdot, 1,<\right)$. All these properties of $\exp _{M}$ can be verified by simple analytic arguments. However, since this is a rather tedious exercise and not very insightful, we refer the reader to Krapp [13, Section 7.2], where all steps of the proof of the following theorem are presented in detail (see [13, Lemma 7.20 and Theorem 7.26]).

Theorem 3.4 The function $\exp _{M}$ defines an exponential on $K_{M}$. Moreover, $\exp _{M}$ extends base 2 exponentiation on $M$, ie $\exp _{M}(n)=2^{n}$ for any $n \in M$.

Remark 3.5 Note that by construction of $\exp _{M}$ via limits of rational $M$-Cauchy sequences, clearly it is $M$-definable.

Theorem 3.4 enables us to consider $\exp _{M}$ as base 2 exponentiation on $K_{M}$. We thus denote $\exp _{M}(x)$ from now on also by $2^{x}$.

Since $2^{x}$ defines an exponential on $K_{M}$, it is, in particular, a continuous map, as the preimage of any open interval in $K_{M}$ under $2^{x}$ is an open subset of $K_{M}$. An exponential polynomial $p$ with integer coefficients is an expression of the form $p\left(x_{1}, \ldots, x_{n}\right)=r\left(x_{1}, \ldots, x_{n}, 2^{x_{1}}, \ldots, 2^{x_{n}}\right)$ for some $r(\vec{x}, \vec{y}) \in \mathbb{Z}[\vec{x}, \vec{y}]$. Note that by Corollary 3.3, any exponential polynomial over $\left(K_{M}, 2^{x}\right)$ is continuous and $M$-definable. Hence, by the conventions introduced at the end of Section 3.1, we can make sense of expressions of the form $\mathbb{Q}_{M} \models \varphi(\vec{a})$, where $\vec{a} \in K_{M}$ and $\varphi$ is an arbitrary $\mathcal{L}_{\exp }-$ formula with quantification over $M, \mathbb{Z}_{M}$ as well as $\mathbb{Q}_{M}$. Note that by the conventions we established, for any quantifier-free $\mathcal{L}_{\text {exp }}$-formula $\varphi$ and any $\vec{a} \in K_{M}$, we have $\mathbb{Q}_{M}=\varphi(\vec{a})$ if and only if $\left(K_{M}, 2^{x}\right)=\varphi(\vec{a})$. 


\section{Model theoretic properties}

In the previous sections, we fixed a model $M \models$ PA and constructed an $M$-definable exponential $2^{x}$ on $K_{M}$. In this section, we first consider models of true arithmetic and prove our main result (see Theorem 4.5), then turn to models of Peano arithmetic and obtain partial results regarding Conjecture 4.6 and finally show that bounded arithmetic does not suffice for our construction of an exponential.

\subsection{True arithmetic}

It was first shown by Wilkie [23] that the theory of real exponentiation $T h\left(\mathbb{R}_{\exp }\right)$ is model complete. As a consequence, $\mathrm{Th}\left(\mathbb{R}_{\mathrm{exp}}\right)$ is axiomatised by all its $\forall_{2}$-sentences ${ }^{5}$ (see eg Hodges [9, Theorem 8.3.3]). Due to the following lemma (see eg Macintyre [17, Section 2.5] or Krapp [13, Lemma 4.3]), an $\mathcal{L}_{\exp }$-structure is a model of $\mathrm{Th}\left(\mathbb{R}_{\exp }\right)$ if and only if it satisfies all sentences in $\operatorname{Th}\left(\mathbb{R}_{\exp }\right)$ which are of the form $\forall \vec{x} \exists \vec{y} p(\vec{x}, \vec{y})=0$ for some exponential polynomial $p$.

Lemma 4.1 Let $\varphi(\vec{u}, \vec{y})=\varphi\left(u_{1}, \ldots, u_{m}, \vec{y}\right)$ be a quantifier-free $\mathcal{L}_{\exp }$-formula. Then for any real closed exponential field $(K, E)$, the formula $\exists \vec{u} \varphi(\vec{u}, \vec{y})$ is equivalent over $(K, E)$ to a formula of the form $\exists \vec{x} p(\vec{x}, \vec{y})=0$ for some exponential polynomial $p(\vec{x}, \vec{y})=p\left(x_{1}, \ldots, x_{n}, \vec{y}\right)$ with integer coefficients (where possibly $n \neq m$ ).

For the next lemma, recall that by the conventions established in Section 3 we have that $M$-reals can also be defined by $\mathcal{L}_{\text {exp }}$-formulas and that, in this regard, by Lemma 2.4 we may assume that all occurring $\mathcal{L}_{\text {exp }}$-formulas are safe formulas.

Lemma 4.2 Let $M \models$ PA and let $r\left(x_{1}, \ldots, x_{k}, y_{1}, \ldots, y_{m}\right)$ be an exponential polynomial with integer coefficients. Moreover, let $\varphi_{i}(n, y, \vec{v})$ be $\mathcal{L}_{\exp }$-formulas. Then there exist $\mathcal{L}_{\text {exp }}$-formulas $\psi_{i}(n, y, \vec{v})$ such that for any $\vec{p} \in \mathbb{Q}_{M}$, we have $\left(K_{M}, 2^{x}\right) \models$ $\exists \vec{y} r\left(x_{\varphi_{1}}^{\vec{p}}, \ldots, x_{\varphi_{k}}^{\vec{p}}, \vec{y}\right)=0$ if and only if:

$$
\mathbb{Q}_{M}=r\left(x_{\varphi_{1}}^{\vec{p}}, \ldots, x_{\varphi_{k}}^{\vec{p}}, x_{\psi_{1}}^{\vec{p}}, \ldots, x_{\psi_{m}}^{\vec{p}}\right)=0
$$

Proof We first assume that $k=m=1$. Let $\vec{p} \in \mathbb{Q}_{M}$ such that $\left(K_{M}, 2^{x}\right) \models \exists y r\left(x_{0}, y\right)=$ 0 , where $x_{0}=x_{\varphi_{1}}^{\vec{p}_{1}}$. Since $r$ is continuous and $\mathbb{Q}_{M}$ is dense in $K_{M}$, we obtain that

$$
\mathbb{Q}_{M} \models \forall(\varepsilon>0) \exists(\delta>0) \forall t\left(\left|t-y_{0}\right|<\delta \rightarrow\left|r\left(x_{0}, t\right)\right|<\varepsilon\right)
$$

\footnotetext{
${ }^{5} \mathrm{An} \forall_{2}$-formula is of the form $\forall \vec{x} \exists \vec{y} \varphi(\vec{x}, \vec{y}, \vec{v})$, where $\varphi$ is quantifier-free (see [9, page 47f]).
} 
where $y_{0} \in K_{M}$ with $r\left(x_{0}, y_{0}\right)=0$. Now consider the formula $\alpha(\vec{p}, L, k, \ell)$ given by:

$$
\forall(\varepsilon>0) \exists q_{1}, q_{2}\left(L+\frac{k}{2^{\ell}} \leq q_{1}<q_{2} \leq L+\frac{k+1}{2^{\ell}} \wedge \forall\left(t \in\left[q_{1}, q_{2}\right]\right)\left|r\left(x_{0}, t\right)\right|<\varepsilon\right)
$$

By (4-1) and density of $\mathbb{Q}_{M}$ in $K_{M}$, the set

$$
A=\left\{L \in \mathbb{Z}_{M}: \mathbb{Q}_{M} \models \forall(\ell \in M) \exists\left(k \in M^{<2^{\ell}}\right) \alpha(\vec{p}, L, k, \ell)\right\}
$$

which is definable over $\mathbb{Q}_{M}$, is non-empty. (For instance, $\left\lfloor y_{0}\right\rfloor \in A$.) Hence, we can choose $L_{0} \in \mathbb{Z}_{M}$ such that $\left|L_{0}\right|$ is minimal. By definition of $A$, for any $n \in M$ we can choose the least $k(n) \in M$ with $k<2^{n}$ such that $\mathbb{Q}_{M} \models \alpha\left(\vec{p}, L_{0}, k(n), n\right)$. Hence, for any $n \in M$

$$
\begin{aligned}
\mathbb{Q}_{M} & =\forall(\varepsilon>0) \exists q_{1}, q_{2} \\
& \left(L_{0}+\frac{k(n)}{2^{n}} \leq q_{1}<q_{2} \leq L_{0}+\frac{k(n)+1}{2^{n}} \wedge \forall\left(t \in\left[q_{1}, q_{2}\right]\right)\left|r\left(x_{0}, t\right)\right|<\varepsilon\right)
\end{aligned}
$$

and $k(n)$ is least with that property. By choice of $k(n)$, we have that $a_{n}=L_{0}+k(n) / 2^{n}$ is a rational $M$-Cauchy sequence, as $\left|a_{n}-a_{n+1}\right| \leq 1 / 2^{n}$. Moreover, the $M$-real $a=\lim _{n \rightarrow \infty} a_{n}$ satisfies $r\left(x_{0}, a\right)=0$, as for any $\varepsilon \in \mathbb{Q}_{M}^{>0}$ we have $\left|r\left(x_{0}, a\right)\right|<\varepsilon$. Finally, let $\psi(n, y, \vec{v})$ be the safe formula given by Lemma 2.4 such that $\psi(n, y, \vec{p})$ defines the $M$-sequence $a_{n}$. Then $\mathbb{Q}_{M}=r\left(x_{0}, x_{\psi}^{\vec{p}}\right)=0$, as required.

Note that the construction of $\psi$ did not depend on the tuple $\vec{p}$. Hence, if $\vec{p} \in \mathbb{Q}_{M}$ such that $\left(K_{M}, 2^{x}\right)=\forall y\left|r\left(x_{0}, y\right)\right|>0$, then the set $A$ in the construction above is empty. By Lemma 2.4, we obtain that $x_{\psi}^{\vec{p}}=0$. Thus, $\mathbb{Q}_{M} \models\left|r\left(x_{0}, x_{\psi}^{\vec{p}}\right)\right|>0$, yielding the required result.

The general case, ie the case that $k, m \geq 1$, works in an analogous way: the absolute values in each formula above can be replaced by an appropriate norm such as the maximum norm. In order to construct a tuple of sequences $\left(k_{1}(n), \ldots, k_{m}(n)\right) \in M^{m}$, one can choose the least element of $M^{m}$, with respect to the lexicographic ordering, satisfying a multivariable version of the formula $\alpha$.

We call the formulas $\left(\psi_{1}, \ldots, \psi_{m}\right)$ in Lemma 4.2 the uniformising formulas for $\left(\varphi_{1}, \ldots, \varphi_{k}\right)$ in $r$. Lemma 4.2 immediately implies the following.

Lemma 4.3 $\left(\forall_{2}\right.$-Uniformisation) Let $M \models$ PA and let $r\left(x_{1}, \ldots, x_{n}, y_{1}, \ldots, y_{m}\right)$ be an exponential polynomial with integer coefficients such that $\left(K_{M}, 2^{x}\right) \models \forall \vec{x} \exists \vec{y} r(\vec{x}, \vec{y})=0$. Then for every $n$-tuple $\left(\varphi_{1}, \ldots, \varphi_{n}\right)$ of $\mathcal{L}_{\exp }$-formulas, the corresponding $m$-tuple $\left(\psi_{1}, \ldots, \psi_{m}\right)$ of uniformising $\mathcal{L}_{\exp }$-formulas satisfies:

$$
\mathbb{Q}_{M}=\forall \vec{v} r\left(x_{\varphi_{1}}^{\vec{v}}, \ldots, x_{\varphi_{n}}^{\vec{v}}, x_{\psi_{1}}^{\vec{v}}, \ldots, x_{\psi_{m}}^{\vec{v}}\right)=0
$$


We now specialise to models of true arithmetic and start with the standard model $\mathbb{N}$.

Lemma 4.4 The real closed exponential field $\left(K_{\mathbb{N}}, 2^{x}\right)$ is a model of real exponentiation.

Proof By Lemma 4.1, it suffices to show that any $\forall_{2}$-sentence in $\operatorname{Th}\left(\mathbb{R}_{\text {exp }}\right)$ whose quantifier-free part is an exponential polynomial equation holds in $\left(K_{\mathbb{N}}, 2^{x}\right)$. Again, we assume for the sake of simplicity that any such formula consists of one universal and one existential quantifier. Let $\forall x \exists y r(x, y)=0 \in \mathrm{Th}\left(\mathbb{R}_{\exp }\right)$ where $r$ is an exponential polynomial with integer coefficients. Moreover, let $\varphi(n, y, \vec{v})$ be an $\mathcal{L}_{\text {exp }}$-formula and let $\vec{p} \in \mathbb{N}$. As Theorem 3.4 implies that $\left(K_{\mathbb{N}}, 2^{x}\right)$ is a substructure of $\mathbb{R}_{\text {exp }}$, we obtain $\mathbb{R}_{\exp }=\exists y r\left(x_{\varphi}^{\vec{p}}, y\right)=0$. Since $\mathbb{Q}_{\mathbb{N}}=\mathbb{Q}$ is dense in $\mathbb{R}$, we can follow the same steps as in the proof of Lemma 4.2 and obtain that the resulting uniformising formula $\psi$ for $\varphi$ satisfies $\left(K_{\mathbb{N}}, 2^{x}\right) \models r\left(x_{\varphi}^{\vec{p}}, x_{\psi}^{\vec{p}}\right)=0$.

Theorem 4.5 Let $M \models \mathrm{Th}(\mathbb{N})$. Then $\left(K_{M}, 2^{x}\right)$ is a model of real exponentiation.

Proof By Lemma 4.4, it suffices to prove that $\left(K_{M}, 2^{x}\right) \equiv\left(K_{\mathbb{N}}, 2^{x}\right)$ and in this regard to show that any $\forall_{2}$-sentence in $\operatorname{Th}\left(K_{\mathbb{N}}, 2^{x}\right)$ also holds in $\left(K_{M}, 2^{x}\right)$. Let $r\left(x_{1}, \ldots, x_{n}, y_{1}, \ldots, y_{m}\right)$ be an exponential polynomial with integer coefficients such that $\left(K_{\mathbb{N}}, 2^{x}\right)=\forall \vec{x} \exists \vec{y} r(\vec{x}, \vec{y})=0$. Let $\left(\varphi_{1}, \ldots, \varphi_{n}\right)$ be a tuple of $\mathcal{L}_{\exp }$-formulas and let $\left(\psi_{1}, \ldots, \psi_{m}\right)$ be their uniformising formulas as in Lemma 4.3. Then $\mathbb{Q} \models$ $\forall \vec{v} r\left(x_{\varphi_{1}}^{\vec{v}}, \ldots, x_{\varphi_{n}}, x_{\psi_{1}}^{\vec{v}_{1}}, \ldots, x_{\psi_{m}}^{\vec{v}_{1}}\right)=0$. By elementary equivalence, we obtain that for any $\vec{p} \in M$,

$$
\mathbb{Q}_{M} \models r\left(x_{\varphi_{1}}^{\vec{p}}, \ldots, x_{\varphi_{n}}^{\vec{p}}, x_{\psi_{1}}^{\vec{p}}, \ldots, x_{\psi_{m}}^{\vec{p}}\right)=0
$$

yielding the required conclusion.

\subsection{Peano arithmetic}

As mentioned in the introduction, it is possible that Theorem 4.5 already holds for models of PA.We highlight this as the following conjecture.

Conjecture 4.6 Let $M \models \mathrm{PA}$. Then $\left(K_{M}, 2^{x}\right)$ is a model of real exponentiation.

In the following, we show that under the assumption of Real Schanuel's Conjecture (SC), any model complete real closed exponential field $\left(K_{M}, 2^{x}\right)$, where $M \models \mathrm{PA}$, satisfies the existential theory $\mathrm{Th}_{\exists}\left(\mathbb{R}_{\exp }\right)$ of real exponentiation. ${ }^{6}$ This conjecture is tightly

\footnotetext{
${ }^{6}$ We thank an anonymous referee for suggesting to study this question under the assumption of Schanuel's Conjecture.
} 
connected to the decidability of the complete theory $\mathrm{Th}\left(\mathbb{R}_{\exp }\right)$ of $\mathbb{R}_{\exp }$ : Macintyre and Wilkie [16] proved that $\mathrm{Th}\left(\mathbb{R}_{\exp }\right)$ is decidable if and only if its existential theory $\mathrm{Th}_{\exists}\left(\mathbb{R}_{\exp }\right)$ is recursively axiomatisable. Moreover, they showed that the latter holds if one assumes (SC). ${ }^{7}$

Real Schanuel's Conjecture (SC) Let $\alpha_{1}, \ldots, \alpha_{n} \in \mathbb{R}$ be linearly independent over $\mathbb{Q}$. Then the transcendence degree of $\mathbb{Q}\left(\alpha_{1}, \ldots, \alpha_{n}, \mathrm{e}^{\alpha_{1}}, \ldots, \mathrm{e}^{\alpha_{n}}\right)$ over $\mathbb{Q}$ is at least $n$.

For the remainder of this section, fix a model $M \models \mathrm{PA}$. Recall that we denote the standard base e exponential on $\mathbb{R}$ by exp. We first show that whenever $\left(K_{M}, 2^{x}\right)$ is model complete, it is definably complete, ie any non-empty $\mathcal{L}_{\text {exp }}$-definable subset of $K_{M}$ which is bounded from below has an infimum in $K_{M}$.

Lemma 4.7 Let $A \subseteq \mathbb{Q}_{M}$ be non-empty and bounded from below and suppose that there is some $\mathcal{L}_{\exp }$-formula $\psi(x, \vec{z})$ and some $\vec{b} \in K_{M}$ such that for any $a \in \mathbb{Q}_{M}$, we have $a \in A$ if and only if $\mathbb{Q}_{M}=\psi(a, \vec{b})$. Then $A$ has an infimum in $K_{M}$.

Proof Let $q_{0} \in \mathbb{Q}_{M}$ be a lower bound of $A$ and let $\psi^{\prime}\left(x, \vec{b}, q_{0}\right)$ be the formula $\exists y\left(\psi(y, \vec{b}) \wedge x>y-q_{0}\right)$. Denote by $A^{\prime}$ the set defined by $\psi^{\prime}\left(x, \vec{b}, q_{0}\right)$, ie $q \in A^{\prime}$ if and only if $\mathbb{Q}_{M}=\psi^{\prime}\left(q, \vec{b}, q_{0}\right)$. Note that all elements of $A^{\prime}$ are strictly positive and $A^{\prime}$ is an upward closed subset of $\mathbb{Q}_{M}$. It suffices to find an infimum $\alpha^{\prime}$ of $A^{\prime}$ in $K_{M}$, as then $\alpha^{\prime}+q_{0}$ is an infimum of $A$. Define the rational $M$-sequence $c_{n} / d_{n}$, where each pair $\left(c_{n}, d_{n}\right) \in M^{2}$ is the least pair, ordered lexicographically, satisfying

$$
\mathbb{Q}_{M} \models \psi^{\prime}\left(\frac{c_{n}}{d_{n}}, \vec{b}, q_{0}\right) \wedge \neg \psi^{\prime}\left(\frac{c_{n}}{d_{n}}-\frac{1}{n}, \vec{b}, q_{0}\right)
$$

ie $c_{n} / d_{n} \in A^{\prime}$ and $c_{n} / d_{n}-1 / n \notin A^{\prime}$. This sequence converges to the $M$-real which is the infimum of $A^{\prime}$ in $K_{M}$.

Proposition 4.8 Suppose that $\left(K_{M}, 2^{x}\right)$ is model complete. Then it is definably complete.

Proof Let $A \subseteq K_{M}$ be a non-empty $\mathcal{L}_{\text {exp }}$-definable set which is bounded from below. By passing to $\left\{x \in K_{M}: \exists(y \in A) x \geq y\right\}$ if necessary (which is definable when $A$ is), we may assume that $A$ is upward closed. Let $A^{\prime}=A \cap \mathbb{Q}_{M}$. Since $\mathbb{Q}_{M}$ is dense in $K_{M}$, the infimum of $A^{\prime}$ in $K_{M}$ is also the infimum of $A$. By Lemma 4.7, it suffices to

\footnotetext{
${ }^{7}$ Macintyre and Wilkie's results are actually on the real exponential field with base e exponentiation, but they hold independently of the base to which exponentiation is carried out.
} 
show that there is an $\mathcal{L}_{\exp }$-formula $\psi(x)$ (with parameters from $K_{M}$ ) such that for any $q \in \mathbb{Q}_{M}$ we have $q \in A^{\prime}$ if and only if $\mathbb{Q}_{M}=\psi(q)$.

Due to the model completeness of $\left(K_{M}, 2^{x}\right)$ and Lemma 4.1, there is an exponential polynomial with integer coefficients $r\left(x_{1}, \ldots, x_{k}, y_{1}, \ldots, y_{m}\right)$ such that $\exists \vec{y} r\left(x_{1}, b_{2}, \ldots, b_{k}, \vec{y}\right)$ $=0$ defines $A$ for some $b_{2}, \ldots, b_{k} \in K_{M}$. Let $\varphi_{i}\left(n, y, v_{0}, \ldots, v_{\ell}\right)$ be $\mathcal{L}_{\exp }$-formulas and let $p_{1}, \ldots, p_{\ell} \in \mathbb{Q}_{M}$ such that for any $p_{0} \in \mathbb{Q}_{M}$ and any $i \in\{2, \ldots, k\}$ we have $b_{i}=x_{\varphi_{i}}^{\left(p_{0}, \ldots, p_{\ell}\right)}$. (For instance, one can choose formulas $\varphi_{i}\left(n, y, v_{0}, \ldots, v_{\ell}\right)$ in which $v_{0}$ does not appear as a free variable.) Moreover, let $\varphi_{1}\left(n, y, v_{0}, \ldots, v_{\ell}\right)$ be the formula $y=v_{0}$. Note that for any $\vec{q}=\left(q_{0}, \ldots, q_{\ell}\right) \in \mathbb{Q}_{M}$ we have $x_{\varphi_{1}}^{\vec{q}}=q_{0}$. Finally, let $\left(\psi_{1}, \ldots, \psi_{m}\right)$ be the uniformising formulas for $\left(\varphi_{1}, \ldots, \varphi_{k}\right)$ in $r$. Then for any $p_{0} \in \mathbb{Q}_{M}$ we have $\left(K_{M}, 2^{x}\right)=\exists \vec{y} r\left(p_{0}, b_{2}, \ldots, b_{k}, \vec{y}\right)=0$ if and only if:

$$
\mathbb{Q}_{M}=r\left(x_{\varphi_{1}}^{\vec{p}}, \ldots, x_{\varphi_{k}}^{\vec{p}}, x_{\psi_{1}}^{\vec{p}}, \ldots, x_{\psi_{m}}^{\vec{p}}\right)=0
$$

Hence, the formula

$$
r\left(x_{\varphi_{1}}^{\left(p_{0}, \ldots, p_{\ell}\right)}, \ldots, x_{\varphi_{k}}^{\left(p_{0}, \ldots, p_{\ell}\right)}, x_{\psi_{1}}^{\left(p_{0}, \ldots, p_{\ell}\right)}, \ldots, x_{\psi_{m}}^{\left(p_{0}, \ldots, p_{\ell}\right)}\right)=0
$$

where $p_{0}$ is considered as the free variable, defines $A^{\prime}$ in $\mathbb{Q}_{M}$.

Next we show unconditionally that $\exp _{M}(x)=2^{x}$ satisfies a differential equation $\exp _{M}^{\prime}=a \exp _{M}$ for some $a \in K_{M}^{>0}$, ie that the following holds in $\left(K_{M}, 2^{x}\right)$ :

$$
\forall(\varepsilon>0) \exists(\delta>0) \forall h\left(0<|h|<\delta \rightarrow\left|\frac{\exp _{M}(x+h)-\exp _{M}(x)}{h}-a\right|<\varepsilon\right)
$$

In order to do so, it is useful to have an element in $K_{M}$ behaving like Euler's number. Consider the sequences:

$$
a_{n}=\left(1+\frac{1}{n}\right)^{n} \text { and } b_{n}=\left(1+\frac{1}{n}\right)^{n+1}
$$

By an easy inductive argument using Bernoulli's inequality, we have that $a_{n}$ is strictly increasing, $b_{n}$ is strictly decreasing and $b_{n}-a_{n}<1 / n$ for any $n \in M^{>0}$. Hence, we can define $\mathrm{e}_{M}$ as $\mathrm{e}_{M}=\lim _{n \rightarrow \infty} a_{n}$.

Lemma 4.9 The inverse functions $\log _{M}$ of $\exp _{M}$ is differentiable in 1 with $\log _{M}^{\prime}(1)=$ $\log _{M}\left(\mathrm{e}_{M}\right)$.

Proof Consider the sequence in $K_{M}$ :

$$
c_{n}=\log _{M}\left(a_{n}\right)=\frac{\log _{M}\left(1+\frac{1}{n}\right)}{\frac{1}{n}}
$$

By the remarks above, $\left(c_{n}\right)$ is strictly decreasing and converges to $\log _{M}\left(\mathrm{e}_{M}\right)$, as required. 
Lemma 4.10 The exponential $\exp _{M}$ is differentiable in 0 with $\exp _{M}^{\prime}(0)=\frac{1}{\log _{M}\left(\mathrm{e}_{M}\right)}$.

Proof By Lemma 4.9, the inverse function of $\exp _{M}$ is differentiable in $\exp _{M}(0)$ with derivative $\log _{M}\left(\mathrm{e}_{M}\right)$. We obtain that $\exp _{M}^{\prime}(0)=\frac{1}{\log _{M}\left(\mathrm{e}_{M}\right)}$.

One can now easily deduce that $\exp _{M}$ is differentiable everywhere with $\exp _{M}^{\prime}=$ $\exp _{M}^{\prime}(0) \exp _{M}$ (see eg Krapp [13, Proposition 2.13]). Set $E(x)=\exp _{M}\left(\log _{M}\left(\mathrm{e}_{M}\right) x\right)$. Then $E$ satisfies the differential equation $E^{\prime}=E$ with initial condition $E(0)=1$. We abbreviate this differential equation by EXP and consider $E$ as base $\mathrm{e}_{M}$ exponentiation.

The above results can be used to show that whenever $\left(K_{M}, \exp _{M}\right)$ is model complete, it is o-minimal. A linearly ordered structure is called o-minimal if any definable subset is a finite union of intervals and points. The study of o-minimal exponential fields whose exponential satisfies EXP has strong links to the decidability of $\mathrm{Th}\left(\mathbb{R}_{\mathrm{exp}}\right)$, as described in Krapp [11].

Proposition 4.11 Suppose that $\left(K_{M}, \exp _{M}\right)$ is model complete. Then it is o-minimal.

Proof By Proposition 4.8, $\left(K_{M}, \exp _{M}\right)$ is definably complete, and by the remarks above, $E$ satisfies EXP. Hence, by Fornasiero and Servi [6] and Hieronymi [8], this implies that $\left(K_{M}, E\right)$ is o-minimal (see Krapp [12, page 7] or [13, Corollary 4.23] for details). Since $\exp _{M}$ is definable in $\left(K_{M}, E\right)$, also $\left(K_{M}, \exp _{M}\right)$ is o-minimal.

Corollary 4.12 Assume (SC) and suppose that $\left(K_{M}, \exp _{M}\right)$ is model complete. Then it satisfies $\mathrm{Th}_{\exists}\left(\mathbb{R}_{\exp }\right)$.

Proof By Servi [20, page 108] under the assumption of (SC), any o-minimal exponential field satisfying EXP is a model of $\mathrm{Th}_{\exists}(\mathbb{R}, \exp )$. Thus, $\left(K_{M}, E\right) \models \mathrm{Th}_{\exists}(\mathbb{R}, \exp )$ by Proposition 4.11. By change of base of exponentiation, we obtain $\left(K_{M}, \exp _{M}\right) \models$ $\mathrm{Th}_{\exists}\left(\mathbb{R}_{\exp }\right)$.

\subsection{A counterexample for bounded arithmetic}

The results of Section 4.1 and Section 4.2 about true arithmetic and Peano arithmetic stand in sharp contrast with the situation for the weaker fragment of bounded arithmetic $\left(I \Delta_{0}\right)$. In this case, already quite weak notions of exponentials may fail to occur. The following definition comes from S. Kuhlmann [15, page 33]. 
Definition 4.13 Let $K$ be a real closed field. An exponential $E$ on $K$ is a GAexponential (where 'GA' stands for 'growth axiom') if, for all $a \in K$ and $n \in \mathbb{N}^{>0}$, we have that $a \geq n^{2}$ implies $E(a)>a^{n}$.

Recall that a non-standard model $M$ of $I \Delta_{0}$ is bounded if there exists a non-standard element $a \in M$ such that $\left\{a^{i}: i \in \mathbb{N}\right\}$ is cofinal in $M$. By the remarks at the beginning of Kaye [10, Chapter 6], such a model can be obtained by taking the set $\left\{x \in N: \exists(i \in \mathbb{N}) x<a^{i}\right\}$, where $N$ is a non-standard model of PA and $a$ is a non-standard element of $N$.

Theorem 4.14 For any bounded non-standard model $M$ of $I \Delta_{0}$ and any real closed field $K$ which has $M$ as an IP, $K$ does not admit a GA-exponential. In particular, no real closed field $K$ which has $M$ as an IP admits a function $g: K \rightarrow K$ such that $(K, g) \equiv \mathbb{R}_{\exp }$.

Proof Let $M$ be a bounded non-standard model of $I \Delta_{0}$, and let $a \in M$ such that $\left\{a^{i}: i \in \mathbb{N}\right\}$ is cofinal in $M$. Assume that $g$ were a GA-exponential on $K$. As $a$ is non-standard, we have $a>n^{2}$ for all $n \in \mathbb{N}$. Hence, we have $g(a)>a^{n}$ for every $n \in \mathbb{N}$. Since $M$ is an IP of $K_{M}$, this implies that $g(a)$ is strictly greater than every element of $K_{M}$, a contradiction.

The second claim is now immediate, as exp is a GA-exponential on $\mathbb{R}$.

\section{Real closures and $M$-compatible exponentials}

In this section, we demonstrate that real closures of models of true arithmetic never support a 'real-like' exponential. This gives an upper limit for the effect that even an IP modelling true arithmetic has on a real closed field. Note that this is not quite surprising, as even considering the standard model $\mathbb{N}$, we get $\mathbb{Q}^{\text {rc }}$ as the real closure of its fraction field, which does not admit an exponential.

Definition 5.1 Let $M=$ PA and let $K$ be a real closed field such that $M$ is an IP of $K$. Then an exponential $E$ on $K$ is $M$-compatible if $\left.E\right|_{M}$ is the usual base 2 exponential on $M$.

Remark 5.2 (1) For any $M=\mathrm{PA}$, the only $M$-compatible exponential on $K_{M}$ is $\exp _{M}$. Indeed, for any $M$-compatible exponential $E$ on $K_{M}$, since $\exp _{M}$ and $E$ agree on $M$, by isomorphism properties they agree on $\mathbb{Q}_{M}$. By continuity of exponentials and density of $\mathbb{Q}_{M}$ in $K_{M}$, they also agree on $K_{M}$. 
(2) For any $M \models \mathrm{PA}$, any $M$-definable exponential $E$ on $K_{M}$ with $E(1)=2$ is $M$-compatible: By isomorphism properties we have $E(n)=2^{n}$ for any $n \in \mathbb{N}$. Since $E$ is $M$-definable, one can then show by induction that $E$ coincides with the standard base 2 exponential on $M$.

To study real closures of models $M$ of $\mathrm{Th}(\mathbb{N})$, we consider how the real closure $\mathbb{Q}_{M}^{\mathrm{rc}}$ of $\mathbb{Q}_{M}$ is coded in $M$.

Definition 5.3 Let $M \models$ PA. An algebraic $M$-real is an $M$-real $r$ such that, for some $p \in \mathbb{Q}_{M}[X]$, we have $p(r)=0$.

Lemma 5.4 For any $d \in \mathbb{N}$ and any $i \in \mathbb{N}^{>0}$, there exists an $\mathcal{L}_{\mathrm{PA}}$-formula $\operatorname{root}_{i}\left(n, y, v_{0}, \ldots, v_{d}\right)$ such that, for every $M \models$ PA and any $a_{0}, \ldots, a_{d} \in \mathbb{Q}_{M}$ such that $f(X)=a_{d} X^{d}+\ldots+a_{1} X+a_{0}$ has at least $i$ distinct zeros in $K_{M}$, the following holds: If $a_{d} \neq 0$, then $\operatorname{root}_{i}(n, y, \vec{a})$ defines an $M$-real $r=x_{\text {root }_{i}}^{\vec{a}}$ such that $f(r)=0$ and there are exactly $i-1$ many $M$-reals below $r$ which are a zero of $f$.

Proof The formula $\operatorname{root}_{i}(n, y, \vec{a})$ is obtained by first finding $L, U \in \mathbb{Z}_{M}$ such that $f$ is positive and increasing or negative and decreasing beyond $U$ and negative and increasing or positive and decreasing before $L$. Note that since $\mathbb{Q}_{M}$ is dense in $K_{M}$ and $f$ is continuous, this can be described by an $\mathcal{L}_{\mathrm{PA}}-$ formula with quantification over $\mathbb{Q}_{M}$. Moreover, by picking $L$ and $U$ such that $|L|,|U| \in M$ are least with that property, $L$ and $U$ are definable over $M$. Now the possible zeros of $f$ must lie in the interval $(L, U)$ in $K_{M}$. By modifying the arguments used in the proof of Lemma 4.2, we can now define exactly the $i$-th zero of $f$ in this interval.

Let us recall the Gelfond-Schneider Theorem (see eg Baker [1, page 10f]).

Theorem 5.5 Let $\alpha, \beta \in \mathbb{R}$ be algebraic such that $\alpha \notin\{0,1\}$ and $\beta \notin \mathbb{Q}$. Then $\alpha^{\beta}$ is transcendental.

Theorem 5.6 Let $M=\operatorname{Th}(\mathbb{N})$. Then $\mathbb{Q}_{M}^{\mathrm{rc}}$ does not admit an $M$-compatible exponential.

Proof Let $R=\mathbb{Q}_{M}^{\mathrm{rc}}$. Assume that $E: R \rightarrow R$ were an $M$-compatible exponential on $R$. Arguing as in Remark 5.2, we obtain $E=\left.\exp _{M}\right|_{R}$.

By Lemma 5.4, the formula $\operatorname{root}_{2}(n, y,-2,0,1)$ defines the positive zero $\sqrt{2}$ of $X^{2}-2$ both in $K_{\mathbb{N}}$ and $K_{M}$. Since $s=\exp _{M}(\sqrt{2})$ is an algebraic $M$-real, we have

$$
\mathbb{Q}_{M} \models \exists b_{0}, \ldots, b_{\ell}\left(b_{\ell} \neq 0 \wedge b_{\ell} s^{\ell}+\ldots+b_{1} s+b_{0}=0\right) .
$$


By elementary equivalence, we obtain that there exists a non-zero polynomial $p(X) \in$ $\mathbb{Q}[X]$ such that $p\left(\exp _{\mathbb{N}}(\sqrt{2})\right)=0$, ie that $2^{\sqrt{2}}$ is algebraic. This contradicts the Gelfond-Schneider Theorem.

We conjecture that the assumptions of Theorem 5.6 can be weakened to $M=\mathrm{PA}$; naturally, our argument depends on whether or not the Gelfond-Schneider Theorem is provable in PA. The question arises whether it is possible for $\mathbb{Q}_{M}^{\text {rc }}$ to admit an exponential which is not necessarily $M$-compatible. In the following, we demonstrate that this is, indeed, the case if $M$ is a countable model of PA. We do so by going through a construction via middle exponentials.

The definition of middle exponentials in ordered fields $K$ is given in S. Kuhlmann [15, Remark 1.20]. For our purposes it is enough to point out that any exponential on the residue field $\bar{K}$ under the natural valuation (ie the finest convex valuation on $K$ ) induces a middle exponential on $K$. Moreover, an exponential on a field $K$ can be constructed by "gluing" a left-, middle- and right-exponential together (see [15, Lemma 1.21]).

Theorem 5.7 Let $K$ be a non-archimedean ordered field and suppose that $K$ has an IP $M$ modelling PA. Then $K$ admits a middle exponential.

Proof By the remarks above, we need to show that the residue field $\bar{K}$ of $K$ under the natural valuation admits an exponential. Since $M$ is an integer part of $K$, we obtain that $\mathbb{Q}_{M}$ is dense in $K$. Hence, the residue fields of $\mathbb{Q}_{M}$ and $K$ coincide. As $\mathbb{Q}_{M}$ is also dense in $K_{M}$, the residue fields of $K$ and $K_{M}$ coincide. Finally, as $K_{M}$ admits an exponential with $\exp _{M}(1)=2$, this induces an exponential on its residue field (see [15, Lemma 1.17]).

Theorem 5.7 gives a positive answer to Berarducci, Ehrlich and S. Kuhlmann [2, page 3316, Question 10] in the countable case. We state it as the following corollary.

Corollary 5.8 Let $K$ be a countable non-archimedean real closed field which has an IP $M$ modelling PA. Then $K$ admits an exponential.

Proof $K$ admits a left-exponential by Carl, D'Aquino and S. Kuhlmann [3], a middleexponential by Theorem 5.7, and a right-exponential by [15, Corollary 1.39].

We conclude this section by presenting an example showing that models of real exponentiation with IPs modelling true arithmetic do not necessarily have an $\mathrm{M}$ compatible exponential. 
Example 5.9 Let $M \models T h(\mathbb{N})$ be non-standard and consider the model of real exponentiation $\left(K_{M}, \exp _{M}\right)$. For this model, $\left.\exp _{M}\right|_{M}$ is the standard base 2 exponential on $M$. By the work of Ressayre [19], for any GA-exponential $E$ on $K_{M}$ which agrees with $\exp _{M}$ on the interval $[0,1]$ in $K_{M}$, we have that $\left(K_{M}, E\right)$ is also a model of real exponentiation. It is now a standard procedure to define a GA-exponential $E$ which agrees with $\exp _{M}$ on the convex hull of $\mathbb{Z}$ in $K_{M}$ (see S. Kuhlmann [15, Section 1.4] and Krapp [13, Section 4] for details): Let $\mathcal{O}$ be the convex hull of $\mathbb{Z}$ in $K_{M}$ and let $\mathbf{A}$ be an ordered group complement to $\mathcal{O}$ in $K_{M}$, ie $K_{M}=\mathbf{A} \oplus \mathcal{O}$ and any element in $\mathbf{A}$ has a non-standard integer part. We define an exponential $E$ on $K_{M}$ by $E(a+b)=\exp _{M}(2 a) \exp _{M}(b)$ for any $a \in \mathbf{A}$ and $b \in \mathcal{O}$. Then for any non-standard element $m \in M$ we have that $E(m) \neq \exp _{M}(m)=2^{m}$. Thus, $E$ is not $M$-compatible. Since $E(x) \geq \exp _{M}(x)$ for any $x \in K_{M}^{>0}$, we obtain by Ressayre's axiomatisation that $\left(K_{M}, E\right) \equiv \mathbb{R}_{\exp }$

\section{Further work}

We conclude this work by motivating and collecting some open questions. Many of the arguments from Section 3 can be extended to any functions that are $M$-definable and preserve the model completeness of $K_{M}$. In particular, it is tempting to apply Gabrièlov's result on the model completeness of the reals with restricted analytic functions (see [7]) and Wilkie's extensions thereof (see [23]).

Question 6.1 Let $M \models \mathrm{Th}(\mathbb{N})$. Under what conditions does $K_{M}$ admit restricted analytic functions $\left(f_{i}\right)$ such that $\left(K_{M},\left(f_{i}\right), \exp _{M}\right)$ is elementarily equivalent to $\mathbb{R}_{\mathrm{an}, \exp }$ (the ordered field of real numbers expanded by all restricted analytic functions as well as exponentiation)?

In Corollary 4.12, we have given a partial answer to Conjecture 4.6. Also in relation to Question 6.1 we would obtain a further strengthening if the assumption of model completeness could be dropped. Again, it may be possible to prove this in a similar way as the model completeness of $\mathbb{R}_{\exp }$ is proved in [23] or van den Dries and Miller [5].

Question 6.2 Let $M=$ PA. Is $\left(K_{M}, \exp _{M}\right)$ model complete?

Furthermore, the general picture regarding subtheories of true arithmetic is still quite rough; it would be nice to refine it by considering further fragments of arithmetic and the impact they have on possible exponentials of real closed fields containing models thereof as IPs. 
$M$ Carl and LS Krapp

\section{Acknowledgements}

During parts of this work, the second author was supported by a doctoral scholarship of Studienstiftung des deutschen Volkes and an Independent Research Grant of Zukunftskolleg, Universität Konstanz.

We thank two anonymous referees for providing numerous useful comments and corrections.

\section{References}

[1] A Baker, Transcendental Number Theory, Cambridge University Press, London (1975)

[2] A Berarducci, P Ehrlich, S Kuhlmann, Mini-Workshop: Surreal Numbers, Surreal Analysis, Hahn Fields and Derivations, Report No. 60/2016, Oberwolfach Rep. 13 (2016) 3313-3372; https://doi.org/10.4171/OWR/2016/60

[3] M Carl, P D'Aquino, S Kuhlmann, On the value group of a model of Peano arithmetic, Forum Math. 29 (2017) 951-957; https://doi.org/10.1515/forum-2015-0226

[4] P D'Aquino, J F Knight, S Starchenko, Real closed fields and models of Peano arithmetic, J. Symb. Log. 75 (2010) 1-11; https://doi.org/10.2178/jsl/1264433906

[5] L van den Dries, C Miller, On the real exponential field with restricted analytic functions, Israel J. Math. 85 (1994) 19-56; https://doi.org/10.1007/BF02758635

[6] A Fornasiero, T Servi, Definably complete Baire structures, Fund. Math. 209 (2010) 215-241; https://doi.org/10.4064/fm209-3-2

[7] A M Gabrièlov, Projections of semianalytic sets, Funkcional. Anal. i Priložen. 2 (1968) no. 4, 18-30 (Russian), Functional Anal. Appl. 2 (1968) 282-291 (English)

[8] P Hieronymi, An analogue of the Baire category theorem, J. Symb. Log. 78 (2013) 207-213; https://doi.org/10.2178/js1.7801140

[9] W Hodges, Model Theory, Encyclopedia of Mathematics and its Applications 42, Cambridge University Press, Cambridge (1993)

[10] R Kaye, Models of Peano Arithmetic, Oxford Logic Guides 15, Oxford Sci. Publ., Oxford Univ. Press, New York (1991)

[11] L S Krapp, O-minimal exponential fields and their residue fields (extended abstract), Oberwolfach Rep. 13 (2016) 3357-3359; https://doi.org/10.4171/OWR/2016/60

[12] L S Krapp, Value groups and residue fields of models of real exponentiation, J. Log. Anal. 11:1 (2019) 1-23; https://doi.org/10.4115/jla.2019.11.1

[13] LS Krapp, Algebraic and Model Theoretic Properties of O-minimal Exponential Fields, Dissertation zur Erlangung des akademischen Grades eines Doktors der Naturwissenschaften (doctoral thesis), Universität Konstanz (2019) 
[14] F-V Kuhlmann, S Kuhlmann, S Shelah, Exponentiation in power series fields, Proc. Amer. Math. Soc. 125 (1997) 3177-3183; https://doi.org/10.1090/S0002-9939-9703964-6

[15] S Kuhlmann, Ordered Exponential Fields, Fields Inst. Monogr. 12 (Amer. Math. Soc., Providence, RI, 2000); https://doi.org/10.1090/fim/012

[16] A Macintyre, A J Wilkie, On the Decidability of the Real Exponential Field, Kreiseliana: about and around Georg Kreisel, ed. P Odifreddi; A K Peters, Wellesley, MA (1996) $441-467$

[17] A Macintyre, Turing meets Schanuel, Ann. Pure Appl. Logic 167 (2016) 901-938; https://doi.org/10.1016/j.apal.2015.10.003

[18] D Marker, J H Schmerl, C Steinhorn, Uncountable real closed fields with PA integer parts, J. Symb. Log. 80 (2015) 490-502; https://doi.org/10.1017/js1.2014.57

[19] J-P Ressayre, Integer Parts of Real Closed Exponential Fields (extended abstract), Arithmetic, Proof Theory and Computational Complexity, Oxford Logic Guides 23, eds. P Clote and J Krajícek; Oxford Sci. Publ., Oxford Univ. Press, New York (1993) 278-288

[20] T Servi, On the First Order Theory of Real Exponentiation, Tesi di Perfezionamento (doctoral thesis), Scuola Normale Superiore di Pisa (2006)

[21] J C Shepherdson, A Non-standard Model for a Free Variable Fragment of Number Theory, Bull. Acad. Polon. Sci. Sér. Sci. Math. Astronom. Phys. 12 (1964) 79-86

[22] S G Simpson, Subsystems of Second Order Arithmetic, 2nd edn, Perspect. Log. (Cambridge University Press, Cambridge 2009); https://doi.org/10.1017/CB09780511581007

[23] A J Wilkie, Model completeness results for expansions of the ordered field of real numbers by restricted Pfaffian functions and the exponential function, J. Amer. Math. Soc. 9 (1996) 1051-1094; https://doi.org/10.1090/S0894-0347-96-00216-0

Institut für mathematische, naturwissenschaftliche und technische Bildung, Europa-Universität Flensburg, 24943 Flensburg, Germany

Fachbereich Mathematik und Statistik, Universität Konstanz,

78457 Konstanz, Germany

merlin.carl@uni-flensburg.de, sebastian.krapp@uni-konstanz.de

http://www . math. uni-konstanz.de/ krapp/

Received: 5 February 2019 Revised: 11 February 2021 